\title{
Teores de mercúrio em cabelo e consumo de pescado de comunidades ribeirinhas na Amazônia brasileira, região do Tapajós
}

\author{
Levels of mercury found in hair and fish consumption of riverine \\ communities in the Tapajós region of the Brazilian Amazon
}

\author{
José Maria Farah Costa Junior ${ }^{1}$ \\ Camile Irene Mota da Silva ${ }^{1}$ \\ Abner Ariel da Silva Lima ${ }^{1}$ \\ Dario Rodrigues Júnior ${ }^{1}$ \\ Luiz Carlos de Lima Silveira ${ }^{1}$ \\ Givago da Silva Souza ${ }^{1}$ \\ Maria da Conceição Nascimento Pinheiro ${ }^{2}$
}

${ }^{1}$ Laboratório de Toxicologia Humana e Ambiental, Núcleo de Medicina Tropical, Universidade Federal do Pará (UFPA). Av. Generalíssimo Deodoro 92, Umarizal. 66055-240 Belém PA Brasil.

farahjunior@hotmail.com. ${ }^{2}$ Programa de

Pós-Graduação em Doenças Tropicais, Núcleo de

Medicina Tropical, UFPA.

Belém PA Brasil.

\begin{abstract}
Riverine communities are exposed to mercury due to the high ingestion of fish in their diet. In order to evaluate the levels of exposure in the Tapajós region, also assessing the fish ingestion frequency, a study was conducted in adults living in riverine communities in the municipality of Itaituba in the State of Pará. Hair samples were collected for the determination of total mercury and the weekly frequency data of fish ingestion was recorded. The mean concentration of total mercury varied from $7.25 \mu \mathrm{g} / \mathrm{g}$ (in 2013) to $10.80 \mu \mathrm{g} / \mathrm{g}$ (in 2014), with no significant difference being observed ( $p=0.1436)$. As for fish ingestion frequency, the majority of the individuals evaluated revealed high consumption both in 2013 and in 2014. High levels of total mercury were observed only in those with high consumption of fish in both years. The importance of ongoing monitoring of exposure levels in humans should be stressed, basing itself on indices of tolerance of $6 \mu \mathrm{g} / \mathrm{g}$ recommended by the World Health Organization, and investigation about the consumption of fish such that strategies for control and prevention are improved.
\end{abstract}

Key words Mercury, Mercury poisoning, Environmental exposure
Resumo Comunidades ribeirinhas estão expostas ao mercúrio devido à dieta composta por elevada ingestão de peixes. Com o objetivo de avaliar os níveis de exposição na região do Tapajós, identificando também a frequência de ingestão de pescado, realizou-se um estudo em adultos residentes nas comunidades ribeirinhas do município de Itaituba/PA. Foram coletadas amostras de cabelo para a determinação de mercúrio total e dados de frequência semanal de ingestão de peixes. A concentração média de mercúrio total variou de

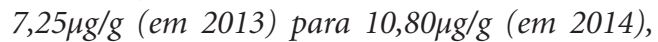
não sendo observada diferença significativa ( $p=$ $0,1436)$. Quanto à frequência de ingestão de pescado, a maioria dos indivíduos avaliados apresentou um alto consumo, tanto em 2013 quanto em 2014. Indices elevados de mercúrio total foram observados somente naqueles com alto consumo de pescado em ambos anos. Ressalta-se a importância da continuação do monitoramento dos niveis de exposição em humanos, fundamentando-se nos indices de tolerância de $6 \mu \mathrm{g} / \mathrm{g}$ preconizado pela Organização Mundial de Saúde, e na investigação quanto ao consumo de peixes para que as estratégias de controle e prevenção sejam melhoradas.

Palavras-chave Mercúrio, Intoxicação por mercúrio, Exposição ambiental 


\section{Introdução}

O mercúrio $(\mathrm{Hg})$ é um elemento químico considerado não essencial a qualquer processo metabólito ${ }^{1}$. Reconhecido por seu potencial tóxico, ele é capaz de bioacumular nos organismos e biomagnificar através da cadeia alimentar ${ }^{2}$.

Uma das vias de exposição ao mercúrio na Amazônia é através da ingestão de alimentos contaminados, mais precisamente o pescado, cuja dieta as comunidades ribeirinhas e pesqueiras utilizam como opção de cardápio alimentar. Segundo Lemire et al. ${ }^{3}$, os peixes representam a alimentação básica e a principal fonte de proteínas para populações tradicionais, as quais merecem atenção especial por possuir maior probabilidade de exposição a níveis considerados perigosos.

Muitos desastres ocorridos no mundo já foram registrados, tanto pela utilização deste metal como pelo consumo de pescado e outros alimentos contaminados. Acidentes de grande amplitude ocorreram na baía de Minamata e Niigata, no Japão; no nordeste do Iraque; na Suécia; na Guatemala; em Alamogordo, no Novo México. Com todos esses casos mencionados, houve a crescente conscientização das consequências que o mercúrio pode causar no ecossistema e na saúde pública ${ }^{4}$.

Estudos na Amazônia, acerca dos níveis de exposição ao $\mathrm{Hg}$, datam desde os anos 90, período o qual altas concentrações foram identificadas, sendo objeto de preocupação em virtude dos riscos a saúde que este metal poderia provocar a populações expostas ${ }^{5-15}$.

Na região do Tapajós, os índices em amostras de cabelos configuram uma exposição permanente, sendo o pescado da região a fonte desta exposição ${ }^{10,11,16}$. A poluição ambiental neste local foi resultante tanto do despejo de mercúrio metálico decorrente da garimpagem de ouro, como de outras fontes que contaminaram os ecossistemas aquáticos, chegando à população humana através da dieta, em sua forma de metilmercúrio ${ }^{17}$.

Com o objetivo principal de avaliar os níveis de exposição na região do Tapajós, identificando também a frequência de ingestão de pescado, é proposto testar a hipótese de que nos anos 2013/2014 houve diferença na concentração de mercúrio e na frequência de ingestão de pescado nas comunidades ribeirinhas do município de Itaituba/PA.

\section{Método}

\section{População alvo/Local de estudo}

Trata-se de um estudo observacional transversal, selecionando somente ribeirinhos adultos (18 a 60 anos), de ambos os sexos, residentes nas comunidades de São Luiz do Tapajós (SLT) e de Barreiras (BAR), ambas situadas no município de Itaituba, considerado município que compõem a região de integração do Tapajós. Este município, historicamente, sofre influência da garimpagem de ouro, estando localizado na região sudoeste do estado do Pará, limitando-se com o estado do Amazonas. Possui como coordenadas geográficas $04^{\circ} 16^{\prime} 34^{\prime \prime}$ de latitude sul, e $55^{\circ} 59^{\prime} 06^{\prime \prime}$ de longitude oeste. A população estimada era de 98.493 habitantes, correspondentes à área urbana e rural, incluindo as comunidades distribuídas às margens do rio Tapajós ${ }^{18}$. Estimada pelo censo da Estratégia de Saúde da Família/MS em 460 habitantes, a comunidade de SLT está situada na margem esquerda do rio Tapajós. E a comunidade de BAR está situada na margem direita à jusante Itaituba, com uma população residente estimada em 830 habitantes. As famílias residentes nessas comunidades vivem da agricultura da mandioca e da pesca de subsistência como o meio de sobrevivência financeira ${ }^{18}$.

\section{Determinação do mercúrio total}

De cada voluntário foi colhido amostras de cabelo para a determinação do mercúrio total (HgT). A amostra foi retirada de três locais diferentes do couro cabeludo, cerca de 1 centímetro de distância de sua inserção. Acondicionadas em envelope de papel devidamente identificados para serem analisadas no laboratório de toxicologia humana e ambiental do núcleo de medicina tropical (NMT), da Universidade Federal do Pará - UFPA ${ }^{19}$.

No laboratório, as amostras de cabelo foram enxaguadas com detergente neutro, lavadas intensamente com água deionizada para retirada do detergente, lavadas com $3 \mathrm{ml}$ de acetona e colocadas para secarem capela de exaustão para serem picotadas até a obtenção da amostra em forma de pó. Através da espectrofotometria de absorção atômica com amalgamação em lâmina de ouro, uma quantidade de $0,1 \mathrm{~g}$ de microfragmentos de cabelo foi submetida a um medidor de mercúrio automático denominado comercialmente como Mercury Analyzer (MA), modelo SP-3D da Nippon Corporation-Japão, tendo os resultados expressos em $\mu \mathrm{g} / \mathrm{g}^{19}$. 
A determinação de HgT seguiu as recomendações do fabricante do equipamento - NIC Corporation, sendo utilizadas igualmente por $\mathrm{Pi}$ nheiro et al. ${ }^{5}$, Khoury et al. ${ }^{12}$, Amoras ${ }^{20}$, Corvelo et al. ${ }^{11}$, Milhomem Filho ${ }^{21}$, Pinheiro et al. ${ }^{13}$, Lima et al. ${ }^{14}$ e Costa Junior et al. ${ }^{15}$.

\section{Frequência de ingestão semanal de pescado}

A frequência de ingestão (refeições) semanal de pescado na dieta foi avaliado através de formulário seguindo a classificação descrito por Brune et al. ${ }^{22}$. $\mathrm{O}$ autor classifica o consumo de peixe semanal em cinco categorias: categoria I, nenhum consumo de peixe; categoria II, $<2$ refeições de peixe/semana; categoria III, 2-4 refeições de peixe/semana; categoria IV, > 4 refeições de peixe/ semana; e categoria V, consumo desconhecido.

Foi considerado para este estudo: categoria III, como consumo moderado de pescado e a categoria IV, como alto consumo de pescado, desprezando a categoria I e II, como baixo consumo e aqueles com consumo desconhecido.

\section{Análise estatística}

Os resultados da concentração de $\mathrm{HgT}$ por serem variáveis quantitativas continuas foram expressas por medidas de tendência central (média aritmética e mediana) e de dispersão (desvio padrão). O teste Mann-Whitney foi utilizado para comparação das medianas de HgT entre os anos (2013-2014).

A frequência de ingestão de pescado foi ajustado através de frequência e percentual, pelo fato das variáveis serem classificadas como qualitativas nominais. Para avaliar diferença na frequência de ingestão de pescado entre os anos foi adotado o teste G. O teste Mann-Whitney foi utilizado para comparação das medianas da concentração de HgT daqueles com alto consumo.

As informações inseridas em banco de dados foram analisadas, adotando $=0,05$ ou $5 \%$, com um intervalo de confiança de 95\%. Para a realização dos testes foi escolhido o pacote estatístico Bio Estat $5.0^{23}$.

\section{Aspectos éticos}

O estudo foi conduzido de acordo com as normas da Resolução 466/2012 do Conselho Nacional de Saúde, Ministério da Saúde, Brasil. Foi aprovado pelo CEP - NMT/UFPA. Projeto financiado pelo Conselho Nacional de Desenvolvimento Cientifico e Tecnológico (CNPq).

\section{Resultados}

Foi avaliado um total de 123 amostras de cabelos e dados de consumo de pescado ( 55 do ano de 2013 e 68 do ano de 2014). As concentrações médias de HgT no Tapajós foram 7,25 $\pm 5,61 \mu \mathrm{g} / \mathrm{g}$ e 10,80 $\pm 10,54 \mu \mathrm{g} / \mathrm{g}$, respectivamente, em 2013 e em 2014. Ao comparar a concentração mediana de HgT apresentado pelas comunidades ribeirinhas, percebeu-se que não houve uma diferença significativa nas concentrações entre os anos analisados ( $\mathrm{p}>0,05)$ (Figura 1).

No que diz respeito à ingestão (refeições) de pescado, a frequência de indivíduos com alto consumo foi superior tanto em 2013 quanto em 2014, sendo representativo, respectivamente, por $41(74,5 \%)$ e $65(95,6 \%)$ ribeirinhos (Figura 2). Ao comparar os anos, foi percebida nas comunidades ribeirinhas diferença significativa na frequência de ingestão ( $\mathrm{p}$ valor $=0,0006$ ).

Ao analisar os níveis de HgT conforme a frequência de ingestão semanal de pescado foi observado que, em 2013, os 14 ribeirinhos com consumo moderado e os 41 com alto consumo apresentaram, respectivamente, concentração média de HgT de 5,56 $\pm 5,13 \mu \mathrm{g} / \mathrm{g}$ e de 7,83 \pm $5,70 \mu \mathrm{g} / \mathrm{g}$. Em contrapartida, em 2014, aqueles com consumo moderado ( 3 ribeirinhos) e alto consumo (65 ribeirinhos) obtiveram concentra-

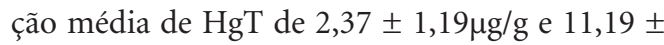
10,62, nesta ordem (Tabela 1).

Houve um aumento tanto na quantidade de indivíduos com alto consumo de pescado quanto na concentração média de HgT destes $(7,83 \mu \mathrm{g} / \mathrm{g}$ para $11,19 \mu \mathrm{g} / \mathrm{g})$, continuando com teores acima do limite ao se basear nos níveis de tolerância de $6 \mu \mathrm{g} / \mathrm{g}$ da Organização Mundial de Saúde (OMS). Comparando a concentração mediana de HgT apresentados por aqueles com alto consumo de pescado, percebeu-se que entre os anos não houve uma diferença significativa $(\mathrm{p}>0,05)$ dos níveis (Figura 3).

\section{Discussão}

Nível de mercúrio no cabelo tem sido amplamente utilizado como um biomarcador confiável para avaliar a exposição humana $a^{5,711-15,20,21,24}$. Neste estudo, foi observado que de 2013 para 2014 a concentração média de mercúrio na região aumentou, permanecendo acima do limite de tolerância $(6 \mu \mathrm{g} / \mathrm{g})$ estabelecido pela OMS. Recentemente, índices acima de $6 \mu \mathrm{g} / \mathrm{g}$ também foram encontrados Khoury et al. ${ }^{12}$, Pinheiro et al. ${ }^{13}$, 


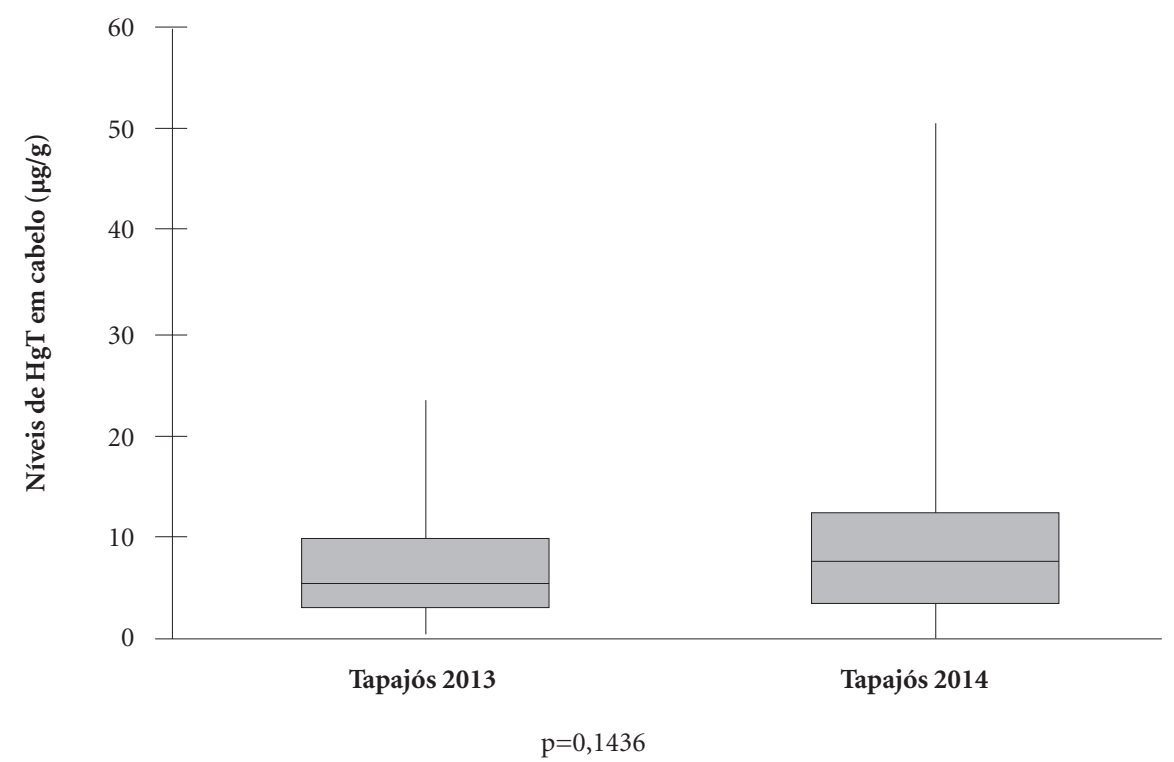

Figura 1. Níveis de HgT em cabelo ( $\mu \mathrm{g} / \mathrm{g}$ ) de ribeirinhos da região do Tapajós, PA, nos anos 2013 e 2014 (teste Mann-Whitney).

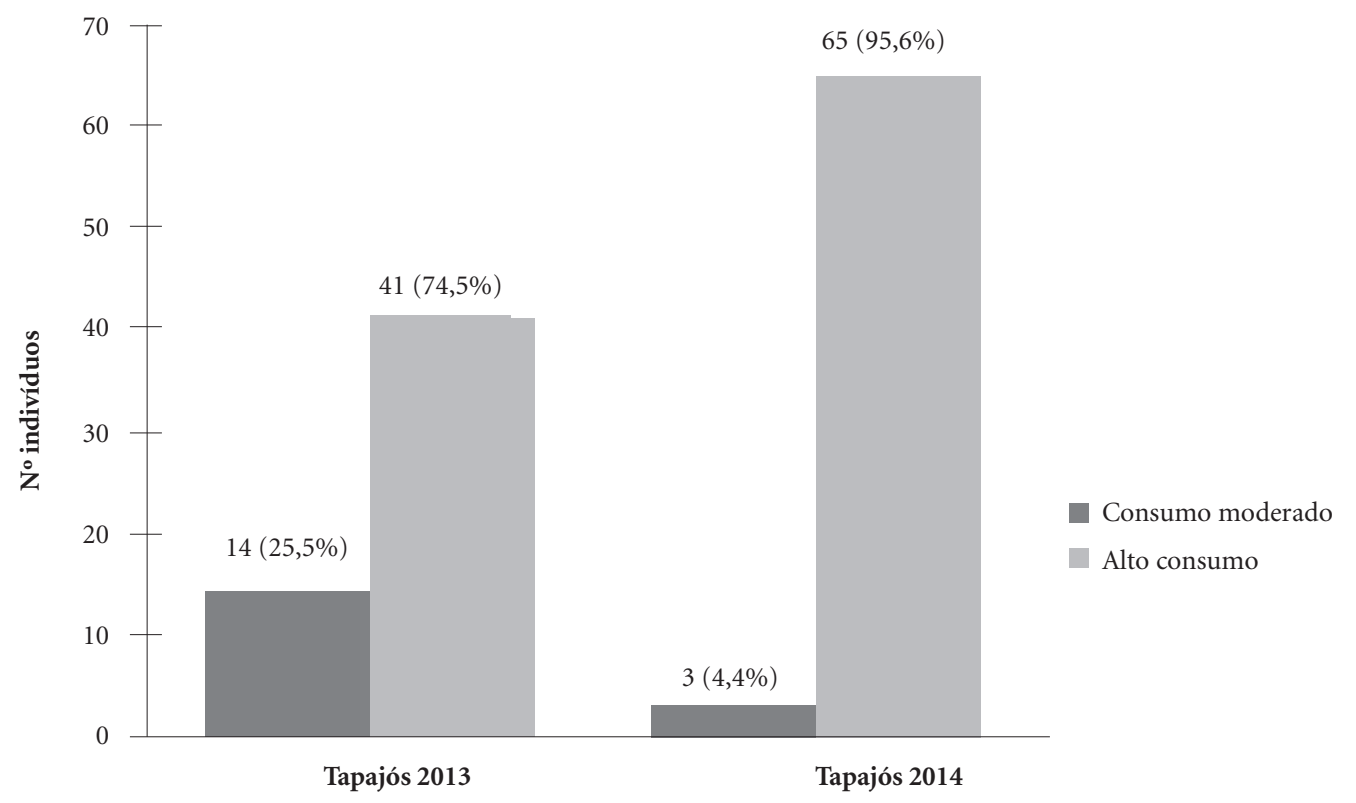

Figura 2. Frequência da ingestão de pescado de ribeirinhos da região do Tapajós, PA, nos anos 2013 e 2014.

Lima et al. ${ }^{14}$, Faial et al. ${ }^{25}$ e por outros autores em estudos anteriores ${ }^{9,10,24,26,27}$.
Níveis elevados podem ser explicados principalmente pela impactação da atividade garim- 
Tabela 1. Níveis de HgT conforme a frequência do consumo de pescado apresentado pelos ribeirinhos da região do Tapajós, PA, nos anos 2013 e 2014.

\begin{tabular}{lcccccc}
\hline \multirow{2}{*}{ Consumo Peixes } & \multicolumn{3}{c}{ Tapajós 2013 } & & & Tapajós 2014 \\
$\mathbf{N}=\mathbf{5 5}$ & & & $\mathbf{N}=\mathbf{6 8}$ & \\
\cline { 2 - 7 } & $\mathbf{n}(\%)$ & $\mathbf{H g T}$ & Md & $\mathbf{n}(\%)$ & $\mathbf{H g T}$ & Md \\
& & $\mathbf{X} \pm \mathbf{D P}$ & & & $\mathbf{X} \pm \mathbf{D P}$ & \\
\hline Consumo moderado & $14(25,5)$ & $5,56 \pm 5,13$ & 3,36 & $3(4,4)$ & $2,37 \pm 1,19$ & 2,26 \\
Alto consumo & $41(74,5)$ & $7,83 \pm 5,70$ & 6,18 & $65(95,6)$ & $11,19 \pm 10,62$ & 7,99 \\
\hline
\end{tabular}

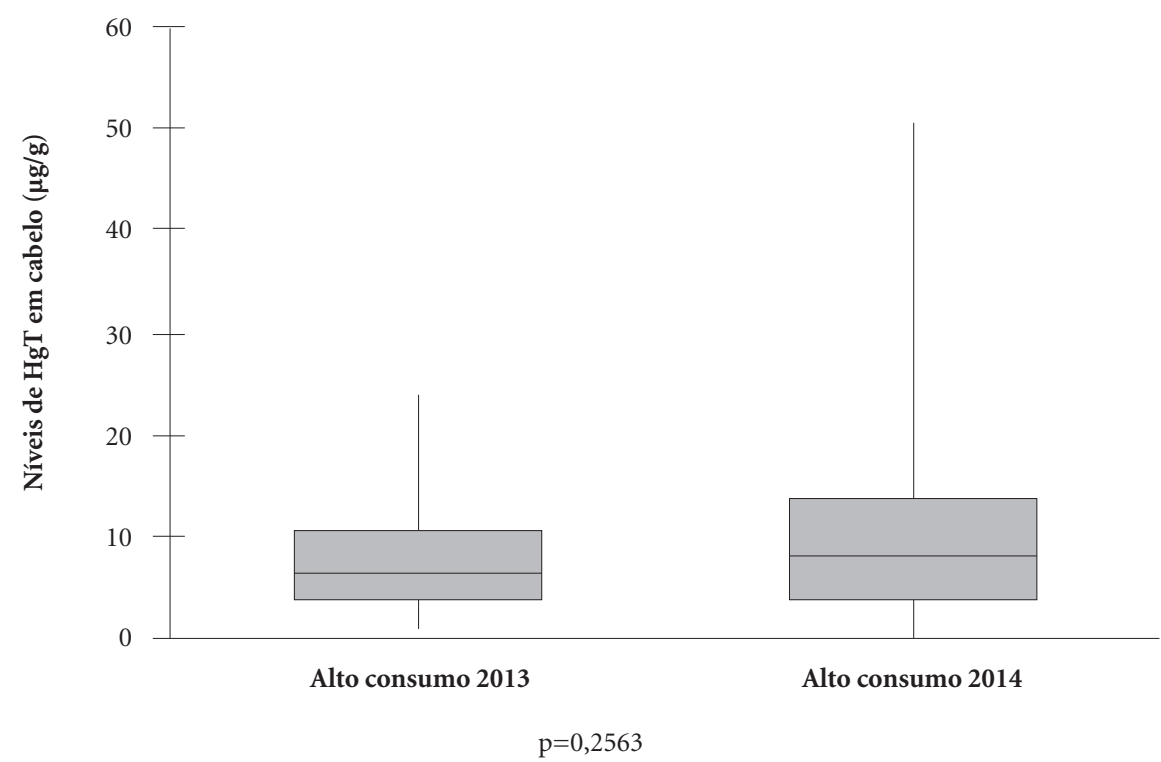

Figura 3. Níveis de HgT em cabelo ( $\mu \mathrm{g} / \mathrm{g}$ ) apresentado por ribeirinhos com alto consumo, nos anos 2013 e 2014 (teste Mann-Whitney).

peira na área com a extração de ouro, resultando na contaminação do ambiente aquático e, consequentemente, no aumento na concentração de mercúrio no pescado, cuja questão precisa ser avaliada, pois a ingestão de peixes é a rota de exposição humana na região do Tapajós ${ }^{10,11,16,17}$. Caso os índices deste metal em peixes contaminados aumentem, maior será o risco de exposição humana. Outra possibilidade seria a falta de variação alimentar na dieta por esta população que segundo Sá et al. ${ }^{28}$ é muito pobre em verduras e legumes, e com um consumo elevado de peixe como principal fonte proteica ${ }^{3,29}$.

Com relação à ingestão de pescado, o número de indivíduos com alto consumo foi superior comparados ao consumo moderado em ambos os anos. Situação também encontrada por Farripas $^{9}$ e Lima et al. ${ }^{14}$ em duas comunidades ribeiri- nhas da região dos Tapajós. O pescado, conforme Bastos $^{30}$, Bastos et al. ${ }^{31}$ e Passos et al. ${ }^{32}$, é a base da dieta alimentar de grande parte da população ribeirinha amazônica, tornando-se assim a principal via de exposição ao Hg para esta população. Outra hipótese para justificar o alto consumo de pescado na região seria o hábito cultural da pesca e da ictiofauna na região do Tapajós, cujos rios fornecem com facilidade a fonte de proteína animal. Defende-se também a questão do isolamento ou o semi-isolamento, no qual as maiorias das populações ribeirinhas vivem que propiciam um mínimo contato social com as cidades mais próximas, favorecendo ao consumo maior de pescado ao comparar com outras fontes alimentares ${ }^{29}$.

Neste estudo, individuos com alto consumo regular de pescado apresentaram maiores concentrações médias de HgT nos anos avaliados, ao 
comparar com aqueles com consumo moderado. Kasper et al. ${ }^{33}$ e Lebel et al. ${ }^{34}$, em seus estudos, afirmam que a quantidade de mercúrio acumulada pelo homem a partir da ingestão do peixe altera em função da quantidade e da frequência com que o peixe é consumido e dos níveis de $\mathrm{Hg}$ presentes nesses peixes. Barbosa et al. ${ }^{35}$ admite que o consumo regular de pescado pelos habitantes do ecossistema amazônico conribui para os altos níveis de contaminação encontrados entre os grupos vulneráveis.

\section{Colaboradores}

JMF Costa Junior participou da interpretação dos dados e redação do artigo. CIM da Silva e AAS Lima participaram das etapas de análise/interpretação dos dados. D Rodrigues Júnior participou da etapa de coleta e análise/interpretação dos dados. LCL Silveira e GS Souza participaram da revisão crítica do artigo. MCN Pinheiro participou da coleta/análise/interpretação dos dados e revisão crítica do artigo.

\section{Conclusão}

Considerando que níveis elevados podem afetar a saúde humana, e que estas concentrações podem ser resultantes dos hábitos alimentares através do consumo de pescado, dos teores de mercúrio nos pescados consumidos e de outras fontes ainda não estudadas como contaminantes dos ecossistemas aquáticos, ressalta-se a importância da continuação do monitoramento dos níveis de exposição em humanos, fundamentando-se nos índices de tolerância de $6 \mu \mathrm{g} / \mathrm{g}$, preconizado pela OMS, e na investigação quanto ao consumo de peixes para que as estratégias de controle e prevenção sejam melhoradas. 


\section{Referências}

1. Tinôco AAP, Azevedo ICAD, Marques EAG, Mounteer AH, Martins CP, Nascentes R, Reis EL, Natalino R. Avaliação de contaminação por mercúrio em Descoberto, MG. Engenharia Sanitária e Ambiental 2010; 15(4):305-314.

2. Araújo BF, Almeida MG, Salomão MSMB, Gobo RR, Siqueira VC, Ovalle ARC, Rezende CE. Distribuição de Hg total e suas associações com diferentes suportes geoquímicos em sedimentos marinhos na margem continental brasileira: Bacia de Campos - Rio de Janeiro. Quim. Nova 2010; 33(3):501-507.

3. Lemire M, Mergler D, Fillion M, Passos CJS, Guimarães JRD, Davidson R, Lucotte M. Elevated blood selenium levels in the Brazilian Amazon. Sci Total Envirion 2006; 366(1):101-111.

4. Roldão AP. Contaminação mercurial em pescado [dissertação]. Rio de Janeiro: Universidade Castelo Branco; 2007.

5. Pinheiro MCN, Guimarães GA, Nakanishi J, Oikawa T, Vieira JL, Quaresma M, Cardoso B, Amoras W. Avaliação da contaminação mercurial mediante análise do teor de $\mathrm{Hg}$ total em amostras de cabelo em comunidades ribeirinhas do Tapajós, Pará, Brasil. Revista da Sociedade Brasileira de Medicina Tropical 2000; 33(2):181184.

6. Santos ECO, Câmara VM, Brabo ES, Loureiro ECB, Jesus IM, Fayal K, Sagica F. Avaliação dos níveis de exposição ao mercúrio entre índios Pakaanóva, Amazônia, Brasil. Cad Saude Publica 2003; 19(1):199-206.

7. Pinheiro MCN. Exposição mercurial e defesas antioxidantes em mulheres ribeirinhas da Amazônia [tese]. Belém: Universidade Federal do Pará; 2005.

8. Santos EO, Jesus IM, Câmara VM, Brabo ES, Jesus MI, Fayal K, Asmus CIRF. Correlation between blood mercury levels in mothers and newborns in Itaituba, Pará State, Brazil. Cad Saude Publica 2007; 23(Supl. 4):622629.

9. Farripas SSM. Aspectos epidemiológicos da exposição ao mercúrio na região do Tapajós, no período de 1994 a 2008 [dissertação]. Belém: Universidade Federal do Pará; 2010.

10. Pinheiro MCN, Farripas SSM, Oikawa T, Costa CA, Amoras WW, Vieira JLF, Silveira AJA, Lima ACM, Souza GS, Silveira LCL. Temporal Evolution of exposure to mercury in riverside communities in the Tapajós Basin, from 1994 to 2010. Bull Environ Contam Toxicol 2012; 89(1):119-124.

11. Corvelo TCO, Oliveira EAF, Parijós AM, Oliveira CSB, Loiola RSP, Araújo AA, Costa CA, Silveira LCL, Pinheiro MCN. Exposição ao mercúrio monitoramento em mulheres em idade reprodutiva que habitam a bacia do Rio Tapajós, Amazônia. Boletim de contaminação ambiental e toxicologia 2014; 93(1):42-46.

12. Khoury EDT, Souza GS, Silveira LCL, Costa CA, Araújo AA, Pinheiro MCN. Manifestações neurológicas em ribeirinhos de áreas expostas ao mercúrio na Amazônia brasileira. Cad Saude Publica 2013; 29(11):2307-2318.

13. Pinheiro MCN, Costa CA, Amoras WW, Silva MCF, Costa Junior JMF, Domingues MM, Santos ECO, Jesus IM, Miranda AMM, Silveira LCL. Manifestações neurológicas em ribeirinhos expostos ao mercúrio na bacia do Tapajós. Rev. Pesq. em Saúde 2015; 14:7-13.
14. Lima ASS, Santos GFS, Silva MCF, Neto EFMP, Costa Junior JMF, Souza GS, Silveira LCL, Pinheiro MCN. Exposição ao mercúrio por meio de dieta de pescado em comunidades ribeirinhas do estado do Pará. Rev. Pesq. em Saúde 2015; 14:22-28.

15. Costa Junior JMF, Lima AAS, Rodrigues Junior D, Silva MCF, Souza GS, Silveira LCL, Pinheiro MCN. Avaliação dos níveis de exposição ao mercúrio em comunidades ribeirinhas da região do Tapajós, no período de 20122014. Rev. Pesq. em Saúde 2015; 14:29-34.

16. Silveira LCL, Ventura DF, Pinheiro MCN. Toxicidade mercurial: avaliação do sistema visual em indivíduos expostos a níveis tóxicos de mercúrio. Ciência e Cultura 2004; 56(1):36-38.

17. Veiga MM, Hinton J, Lilly C. "Mercury in the Amazon: a comprehensive review with special emphasis on bioaccumulation and bioindicators". In: Proceedings of the National Institute for Minamata Disease Forum, Minamata, Japan. October, 1999: 19-39.

18. Instituto Brasileiro de Geografia e Estatística (IBGE). Cidades@ [acessado 2015 Out 4]. Disponível em: http://www.cidades.ibge.gov.br/xtras/home.php.

19. Nippon Instruments Corporation (NIC). Instruction Manual for Mercury SP-3D. Tokio: NIC; 1997.

20. Amoras WW. Interferentes bio-sócio-ambientais na exposição ao mercúrio em crianças ribeirinhas de diferentes regiões da Amazônia [tese]. Belém: Universidade Federal do Pará; 2011.

21. Milhomem Filho EO. Avaliação da exposição ao mercúrio em famílias de pescadores em Imperatriz, Maranhão [dissertação]. Belém: Universidade Federal do Pará; 2012.

22. Brune D, Nordberg GF, Vesterberg O, Gerhardsson L, Wester PO. A review of normal concentrations of mercury in human blood. Sci Total Environ 1991; 100(Spec. n.):235-282.

23. Ayres M, Ayres Junior M, Ayres DL, Santos AAS. Biostat 3.0: aplicações estatísticas nas áreas das ciências biológicas e Médicas. Belém: Sociedade Civil Mamirauá; 2003.

24. Pinheiro MCN, Oikawa, T, Vieira JLF, Gomes MSV, Guimarães GDA, Crespo-López ME, Muller RCS, Amoras WW, Ribeiro DRG, Rodrigues AR, Côrtes MIT, Silveira LCL. Comparative study of human exposure to mercury in riverside communities in the Amazon region. Braz J Med Biol Res 2006; 39(3):411-414.

25. Faial K, Deus R, Deus S, Neves R., Jesus I, Santos E, Alves CN, Brasil D. Mercury levels assessment in hair of riverside inhabitants of the Tapajós River, Pará State, Amazon, Brazil: Fish consumption as a possible route of exposure. J Trace Elem Med Biol 2015; 30:66-76.

26. Schulz AR. Variações interindividuais em biomarcadores de exposição ao mercúrio em uma população ribeirinha do rio Tapajós, Pará [dissertação]. Ribeirão Preto: Universidade de São Paulo; 2009.

27. Dorea J, Barbosa A, Ferrari Í, De Souza J. Mercury in hair and fish consumed by riparian women of the Rio Negro, Brazil. Int J Environ Health Res 2003; 13(3):239248.

28. Sá ALD, Herculano AM, Pinheiro MCN, Silveira LCDL, Nascimento JLMD, Crespo-López ME. Exposição humana ao mercúrio na região Oeste do Estado do Pará. Revista Paraense de Medicina 2006; 20(1):19-25. 
29. Vieira JLF, Gomes ALS, Santos JPN, Lima TCD, Freitas Júnior JA, Pinheiro MCN. Mercury distribution in organs of two species of fish from Amazon region. Bulletin of Environmental contamination and toxicology 2011; 87(4):377-380.

30. Bastos WR. Ocorrência ambiental do mercúrio e sua presença em populações ribeirinhas do Baixo Rio Madeira - Amazônia [tese]. Rio de Janeiro: Universidade Federal do Rio de Janeiro; 2004.

31. Bastos WR, Rebelo MF, Fonseca MF, Almeida R, Malm O. Um estudo descritivo do mercúrio em peixes da bacia do Rio Madeira, Amazônia, Brasil. Act Amaz 2008; 38(3):431-438.

32. Passos CJS, Da Silva DS, Lemire M, Fillion M, Guimarães JRD, Lucotte M, Mergler D. Daily mercury intake in fish-eating populations in the Brazilian Amazon. Journal of Exposure Science and Environmental Epidemiology 2008; 18(1):76-87.

33. Kasper D, Botaro D, Palermo EFA, Malm O. Mercúrio em peixes-fontes e contaminação. Oecologia Brasiliensis 2007; 11(2):228-239.

34. Lebel J, Roulet M, Mergler D, Lucotte M, Larribe F. Fish diet and mercury exposure in a riparian Amazonian population. Water, Air, and Soil Pollution 1997; 97(12):31-44.

35. Barbosa AC, Silva SRL, Dórea JG. Concentration of mercury in hair of indigenous mothers and infants from the Amazon basin. Archives of environmental contamination and toxicology 1998; 34(1):100-105.

Artigo apresentado em 05/04/2016

Aprovado em 08/06/2016

Versão final apresentada em 10/06/2016 